\title{
Spectroscopic and theoretical investigation of the conformational space of a pyrazolo-thiazole precursor of extended dipole diazafulvenium methide intermediates
}

\author{
Cláudio M. Nunes, Susy Lopes, Teresa M.V.D. Pinho e Melo, Rui Fausto* \\ Department of Chemistry, University of Coimbra, Rua Larga, P-3004-535 Coimbra, Portugal
}

\section{A R T I C L E I N F O}

Article history:

Received 4 December 2008

Accepted 19 December 2008

Available online 30 December 2008

\section{Keywords:}

Dimethyl 2,2-dioxo-1H,3H-Pyrazolo[1,5-

c][1,3]thiazole-6,7-dicarboxylate

Matrix isolation

Low temperature FTIR spectroscopy

DFT calculations

Conformational analysis

\begin{abstract}
A B S T R A C T
The structure, preferred conformers and vibrational spectra of the pyrazolo-thiazole precursor of extended dipole diazafulvenium methide intermediates, dimethyl 2,2-dioxo-1H,3H-pyrazolo[1,5c][1,3]thiazole-6,7-dicarboxylate (DPTD) were investigated in low-temperature noble gas matrices (Ar, $\mathrm{Xe}$ ), low temperature neat amorphous and crystalline phases and in $\mathrm{KBr}$ pellet (crystal and melted phases) by infrared spectroscopy, supported by quantum chemical calculations. Two types of conformers were observed spectroscopically in the matrices and in the neat amorphous solid resulting from fast condensation of the vapour of the compound onto the cold (20 K) substrate of the cryostat. These conformers correspond to the two pairs of nearly degenerated structures exhibiting skew/cis (conformers $\mathrm{S}^{\prime} \mathrm{C}$ and $\mathrm{SC}^{\prime}$ ) and gauche/trans (conformers $\mathrm{G}^{\prime} \mathrm{T}$ and $\mathrm{GT}^{\prime}$ ) arrangements of the $\mathrm{N}=\mathrm{C}-\mathrm{C}=\mathrm{O} / \mathrm{C}=\mathrm{C}-\mathrm{C}=\mathrm{O}$ moieties. In the crystalline phase, the vibrational signature of the compound indicates that it exists in a skew/cis arrangement. After melting of the crystal, a conformational mixture is formed, where both skew/cis and gauche/ trans forms exist in equilibrium and, with all probability, also conformer $G^{\prime} C^{\prime}$. This latter conformer cannot exist in the low temperature matrices and amorphous state, in view of the very low energy barrier that separates this form from the lower energy SC' conformer, which can be easily surpassed during deposition of the compound (conformational cooling).
\end{abstract}

(C) 2008 Elsevier B.V. All rights reserved.

\section{Introduction}

Heterocyclic compounds play a central role in chemistry. They have a wide range of applications and represent about half of the known organic compounds [1]. The discovery of new heterocyclic systems and understand their properties and structure are thus extremely important research topics. We have been studying pericyclic reactions of extended dipoles, such as azafulvenium methides and diazafulvenium methides, in order to evaluate the scope of the use of these intermediates in the synthesis of heterocyclic compounds [2-7]. In particular, diazafulvenium methides $\mathbf{2 a - 2 d}$ participate in $[8 \pi+2 \pi]$ cycloadditions giving pyrazolo-annulated heterocycles 3 and undergo intramolecular sigmatropic $[1,8] \mathrm{H}$ shifts affording vinyl- $1 H$-pyrazoles 4-5, some of which with potential biological activity (Scheme 1) [6,7]. The diazafulvenium methides $\mathbf{2 a - 2 d}$ can be generated from the thermal extrusion of sulfur dioxide from 2,2-dioxo-1H,3H-pyrazolo[1,5-c][1,3]thiazoles 1a1d, making relevant the gathering of information on the chemistry and structure of 2,2-dioxo- $1 H, 3 H$-pyrazolo[1,5-c][1,3]thiazoles.

\footnotetext{
* Corresponding author. Tel.: +351 239 852080; fax: +351 239827703 .

E-mail address: rfausto@ci.uc.pt (R. Fausto).
}

The pyrazolo[ $1,5-c][1,3]$ thiazoles belong to a class of heterocycles that possess two fused rings, a pyrazole and a thiazolidine ring, which share a common nitrogen atom. The synthesis of the first derivatives was reported recently by Storr et al. [8-9]. On the other hand, pyrrolo[1,2-c][1,3]thiazoles analogues (a pyrrole and a thiazolidine fused ring that share the same nitrogen atom) are known at least since 1980 and some representatives show interesting biological activities [10-16].

The molecular properties of pyrazolo and pyrrolo-thiazoles are practically unknown. To the best of our knowledge, only our recent study on dimethyl 2,2-dioxo-5-methyl-1H,3H-pyrrolo[1,2c][1,3]thiazole-6,7-dicarboxylate has been reported hitherto [17]. In that study, the conformational space of the compound was investigated using low temperature infrared spectroscopy (for the matrix-isolated monomers, as well as for the compound in the neat amorphous solid and crystalline state) and computational methods [17]. In this study, the conformational space of dimethyl 2,2-dioxo-5-methyl-1H,3H-pyrazolo[1,5-c][1,3]thiazole-6,7-dicarboxylate 1a (DPTD) was investigated using a similar approach in order to set further insight on the structural and spectroscopic properties of this type of extended dipole diazafulvenium methide intermediates precursor. 


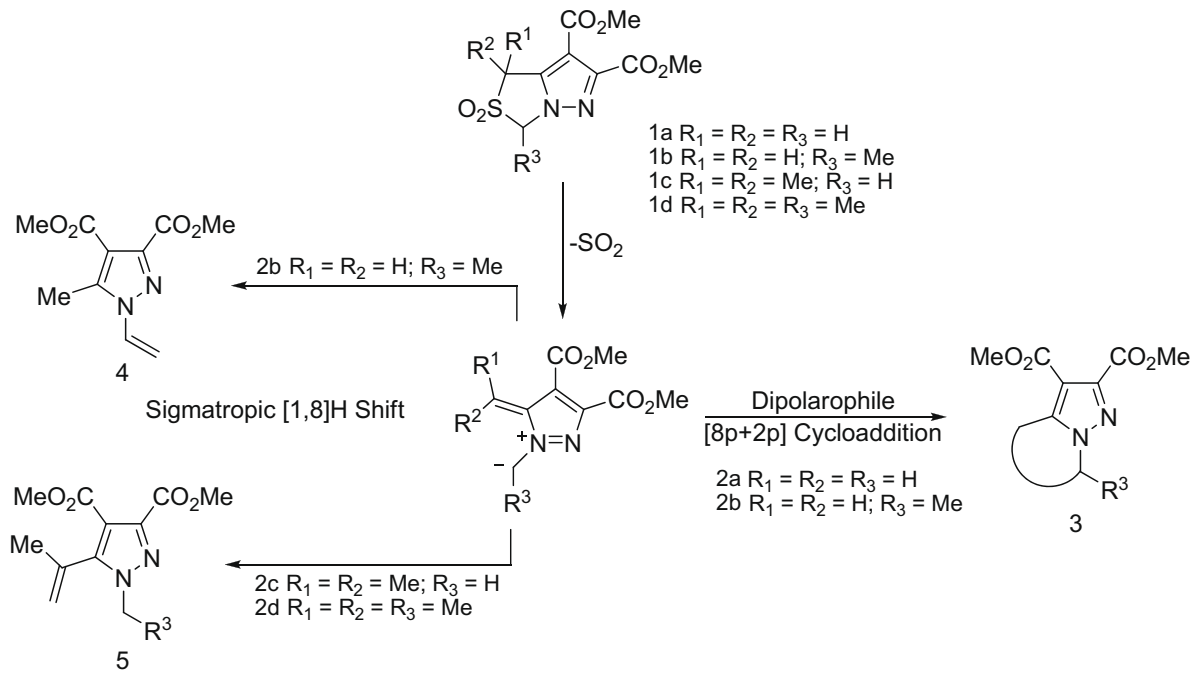

Scheme 1. Reactivity of diazafulvenium methides (2a-2d) generated from thermal extrusion of sulfur dioxide from 2,2-dioxo-1H,3H-pyrazolo[1,5-c][1,3]thiazoles (19a-1d)

\section{Experimental}

\subsection{Synthesis}

Dimethyl 2,2-dioxo-1H,3H-pyrazolo[1,5-c][1,3]thiazole-6,7dicarboxylate (DPTD) 1a was prepared using a known synthetic procedure (Scheme 2) [9]. Thiazolidine-4-carboxylic acid 6, obtained for condensation of $L$-cysteine and formaldehyde in aqueous solution, was nitrosated under standard conditions to give $N$-nitrosothiazolidine-4-carboxylic acid 7. Treatment of 7 with TFAA (trifluoroacetic anhydride) in anhydrous ether furnished the bicyclic mesoionic ring sydnone $\mathbf{8}$ in good yield. This later underwent a $4 \pi+2 \pi$ cycloaddition with dimethyl acetylenedicarboxylate (DMAD) to give the fused pyrazolo[1,5-c][1,3]thiazole 9 in high yield. Finally, oxidation with MCPBA ( $m$-cloroperoxybenzoic acid) gives the sulfone 1a in almost quantitative yield.

\subsection{Infrared spectroscopy}

For matrix isolation and low temperature studies on neat DPDT, the IR spectra were recorded using a Mattson Infinity (60AR series) or Nicolet 6700 Fourier transform infrared spectrometer, equipped with a deuterated triglycine sulphate (DTGS) detector and a $\mathrm{Ge} / \mathrm{KBr}$ beam splitter, with $0.5 \mathrm{~cm}^{-1}$ spectra resolution. Infrared studies at high temperature (298-418 K) were undertaken for the compound as a potassium bromide pellet using a Specac temperature variation cell in a BOMEM (MB40) spectrometer, which has a $\mathrm{Zn} / \mathrm{Se}$ beam splitter and a DTGS detector, with $4 \mathrm{~cm}^{-1}$ spectral resolution. To avoid interference from atmospheric $\mathrm{H}_{2} \mathrm{O}$ and $\mathrm{CO}_{2}$, a stream of dry nitrogen continuously purged the optical path of the spectrometers.

To obtain the matrix spectra, DPTD was sublimated using a specially designed mini-furnace, which was placed inside the cryostat, and co-deposited with a large excess of the matrix gas (argon N60 or xenon N48; both obtained from Air Liquid) onto the CsI optical substrate of the cryostat cooled to $10 \mathrm{~K}$ (for argon matrices) or $20 \mathrm{~K}$ (for xenon matrices). The sublimation temperatures used were $\sim 383 \mathrm{~K}$, in xenon experiments and $\sim 403 \mathrm{~K}$, in argon and neat DPTD amorphous/crystalline state experiments. After depositing of the compound, annealing experiments were performed by increasing the temperature up to $35 \mathrm{~K}$ for, Ar matrices, and $75 \mathrm{~K}$, for Xe matrices. The spectra of the neat amorphous solid of DPTD was obtained using a similar procedure, but with the flux of the cryogenic gas cut off. The solid layer was then allowed to anneal at a slowly increasing temperature up to $298 \mathrm{~K}$. Infrared spectra were collected during this process every $10-20 \mathrm{~K}$ of temperature change. After temperature reached $298 \mathrm{~K}$, the substrate was cooled back to $20 \mathrm{~K}$, and spectra were again collected each $10-20 \mathrm{~K}$.

In all experiments performed, an APD Cryogenics closed-cycle helium refrigeration system with a DE-202A expander was used and the temperature was measured directly at the sample holder by a silicon diode temperature sensor, connected to a digital controller (Scientific Instruments, model 9650-1), with an accuracy of $0.1 \mathrm{~K}$.

\subsection{Computational methodology}

The quantum chemical calculations were performed with Gaussian 03 [18] at the DFT level of theory, using the standard 6$31+G(d)$ and $6-311++G(d, p)$ basis sets [19], and the three-parameter density functional B3LYP, which includes Becke's gradient exchange correction [20] and the Lee, Yang and Parr correlation functional [21]. The conformational space of the DPTD molecule were examined through a systematic independent variation of the two conformationally relevant dihedrals $\left(\mathrm{C}_{2} \mathrm{C}_{1} \mathrm{C}_{14}=\mathrm{O}_{16}\right.$ and $\mathrm{N}_{28} \mathrm{C}_{4} \mathrm{C}_{15}=\mathrm{O}_{18}$; see Fig. 1) from $-180^{\circ}$ to $180^{\circ}$, with increments of $15^{\circ}$. Optimization of the input structures was initially done at the $\mathrm{B} 3 \mathrm{LYP} / 6-31+\mathrm{G}(\mathrm{d})$ level of theory, the minimum energy struc-

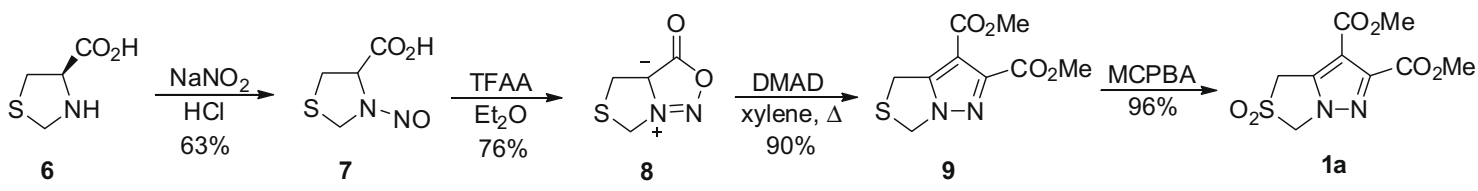

Scheme 2. Synthesis of DPTD (1a). 


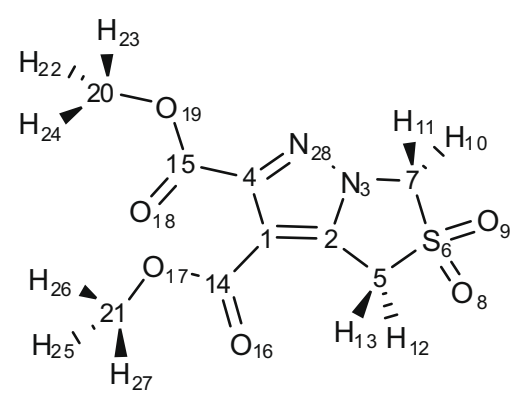

Fig. 1. Adopted numbering scheme for the DPTD molecule. Carbon atoms are represented by their numbers.

tures being subsequently re-optimized and the vibrational frequencies calculated at the B3LYP level with the $/ 6-311++G(d, p)$ split-valence triple- $\zeta$ basis set.

The underestimation of the theoretically predicted frequencies for $S=0$ stretching vibrations has been reported for different types of compounds when calculated at the DFT (B3LYP) level with the 6$311++G(d, p)$ basis set [22-25]. On the other hand, a good agreement between experimental and predicted frequencies was observed for both dimethyl sulphite [23] and sulphate [24] when the B3LYP method was used with the aug-cc-pVQZ augmented split valence quadruple- $\zeta$ basis set, whereas for 3-chloro-1,2-benzisothiazole-1,1-dioxide an accurate reproduction of the frequencies associated with the $S=0$ bond stretching modes could also be achieved at the B3LYP/6-311++G(3df,3pd) level [25]. Indeed it has been noticed that an extensive set of polarization functions is necessary to correctly reproduce frequencies of hypervalent $\mathrm{S}=0$ bonds, at least when the B3LYP functional is used [25]. Since the size of the DPTD molecule makes calculations with an extensive set of polarization functions unpractical the following strategy was used in the present study to optimize the calculated values for the $S=0$ stretching modes in the DPTD molecule: firstly, the vibrational frequencies of these modes calculated at the B3LYP/6311++G(3df,3pd) level for 3-chloro-1,2-benziothiazole-1,1-dioxide [25] were compared to those obtained using the smallest B3LYP/6$311++G(d, p)$ basis set; then, a proper scaling factor was obtained taking into account the frequency ratios [B3LYP/6-311++G(d,p) to B3LYP/6-311++G(3df,3pd)] for the antisymmetric and symmetric $\mathrm{S}=\mathrm{O}$ stretching vibrations (1.046 and 1.051 , respectively); the mean value (1.048) was used as scaling factor for the B3LYP/6$311++G(d, p) S=0$ stretching frequencies calculated for DPTD. For all other frequencies, a scaling factor of 0.990 was used.

Transition state structures and energy barriers for conformational interconversion were obtained using the synchronous transit-guided quasi-Newton (STQN) method [26]. Normal coordinate analysis was undertaken in the internal coordinates space, as described by Schachtschneider [27], using the program BALGA [28] and the optimized geometries and harmonic force constants resulting from the DFT(B3LYP)/6-311++G(d,p) calculations. The internal coordinates used in this analysis were defined as recommended by Pulay et al. [29].

\section{Results and discussion}

\subsection{Geometries and energies}

The DPTD molecule consists of two adjacent ester groups bound to positions 6 and 7 of the 2,2-dioxo- $1 H, 3 H$-pyrazolo[1,5c] $[1,3]$ thiazole nucleus $\left(C_{1}\right.$ and $C_{4}$ in the adopted numbering scheme; see Fig. 1). These esters groups shall adopt a cis arrangement $\left(\mathrm{CH}_{3}-\mathrm{O}-\mathrm{C}=\mathrm{O}\right.$ approximately $\left.0^{\circ}\right)$ since the preference for the cis orientation around the $\mathrm{C}-\mathrm{O}$ bond has been found to repre- sent a general rule for molecules bearing the carboxylic acid or carboxylic ester groups [30-34]. The DPTD molecule can then be conformationally characterized by two internal rotation axes (rotations around the $C_{4}-C_{15}$ and $C_{1}-C_{14}$ bonds), which can be expected to give rise to different low energy conformers. Internal rotations around the $\mathrm{O}_{19}-\mathrm{C}_{20}$ and $\mathrm{O}_{17}-\mathrm{C}_{21}$ bonds would lead to the methyl ester groups adopting high energy trans conformations (over $20 \mathrm{~kJ} \mathrm{~mol}^{-1}$ above the most stable cis forms), which can be safely assumed to be irrelevant in practical terms. The identification of the low energy conformers of DPTD can then be made by systematic investigation of the DFT(B3LYP)/6-31+G(d) potential energy surface (PES) of the molecule, through incremental variation of the two conformationally relevant dihedral angles $\left(\mathrm{C}_{2} \mathrm{C}_{1} \mathrm{C}_{14}=\mathrm{O}_{16}\right.$ and $\mathrm{N}_{28} \mathrm{C}_{4} \mathrm{C}_{15}=\mathrm{O}_{18}$ ) in steps of $15^{\circ}$, with all remaining geometric parameters being optimized at each point. Fig. 2 presents a contour map of the PES calculated as described above. Six energy minima $\left(S^{\prime} C, S^{\prime}, G^{\prime} C^{\prime}, G C, G^{\prime} T\right.$ and $\left.G^{\prime}\right)$ were found on this PES, all belonging to the $C_{1}$ symmetry point group. The geometries of these conformers reoptimized at the DFT(B3LYP)/6-311++G(d,p) level of theory are shown in Fig. 3 (the optimized geometrical parameters for all structures are provided as Supporting Information; Table S1). The calculated relative energies of the six minima as well as the relative energies of these structures with zero-point vibrational energy corrections are given in Table 1.

All studied minimum energy conformations have a planar pyrazole ring and an envelope-like geometry for the thiazolidine ring (with the $\mathrm{C}_{5}, \mathrm{C}_{2}, \mathrm{~N}_{3}$, and $\mathrm{C}_{7}$ atoms nearly in the same plane and the sulphur atom in the apex position, similarly to what was previously found for dimethyl 2,2-dioxo-5-methyl-1H,3H-pyrrolo[1,2c][1,3]thiazole-6,7-dicarboxylate[17]).

The differences in the geometrical parameters calculated for the various minima do not in general exceed $0.6 \mathrm{pm}$ for the bond lengths (except for carbon oxygen bonds, in which the difference can be of $1.9 \mathrm{pm}$ ) or $6-7^{\circ}$ for the bond angles (again with the exception of bond angles of the ester groups). In these forms, the two conformationally relevant dihedral angles $\left[\mathrm{N}_{28} \mathrm{C}_{4} \mathrm{C}_{15}=\mathrm{O}_{18}\right.$ (Phi) and $\mathrm{C}_{2} \mathrm{C}_{1} \mathrm{C}_{14}=\mathrm{O}_{16}$ (Psi)] adopt only two different orientations. Phi assumes absolute values of $\sim 60^{\circ}$ and $\sim 133^{\circ}$, here designated as gauche and skew, and Psi absolute values of $\sim 10^{\circ}$ and $\sim 170^{\circ}$, designated as cis and trans. According to the orientation of these two

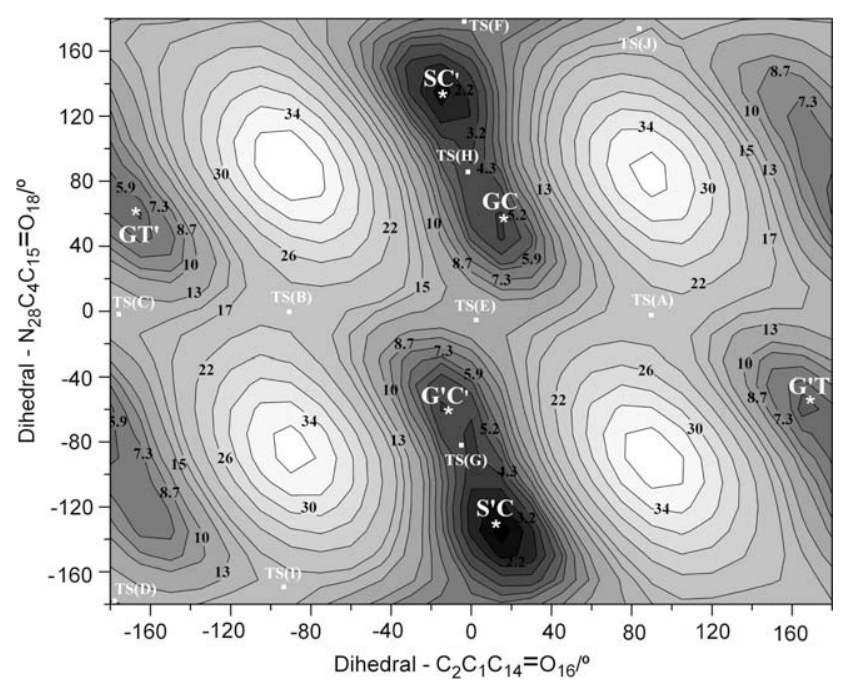

Fig. 2. DFT(B3LYP)/6-31+G(d) potential energy $\left(\mathrm{kJ} \mathrm{mol}^{-1}\right)$ contour map for DPTD as a function of internal rotation about $\mathrm{N}_{28} \mathrm{C}_{4} \mathrm{C}_{15}=\mathrm{O}_{18}$ and $\mathrm{C}_{2} \mathrm{C}_{1} \mathrm{C}_{14}=\mathrm{O}_{16}$ dihedral angles. The two dihedral angles were changed incremently in steps of $15^{\circ}$, and all the remaining coordinates were fully optimized at each point. Minima are indicated by an asterisc and the first-order saddle points by a white square. 


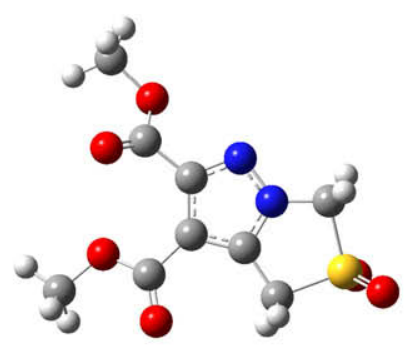

$$
\begin{gathered}
\mathbf{S}^{\prime} \mathbf{C}(0.0) \\
D_{\text {Phi }}=-133.3^{\circ} ; D_{\text {Psi }}=15.7^{\circ}
\end{gathered}
$$

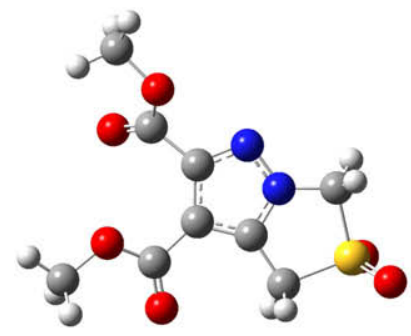

SC' $(0.63)$

$D_{\text {Phi }}=132.1^{\circ} ; D_{\text {Psi }}=-14.1^{\circ}$

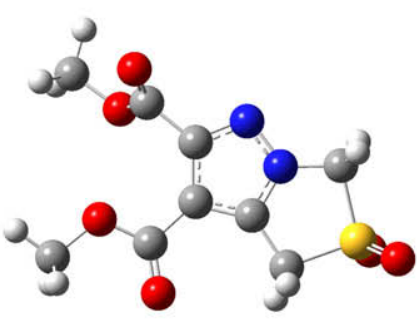

G'C' (2.08)

$D_{\text {Phi }}=-66.1^{\circ} ; D_{\text {Psi }}=-6.9^{\circ}$

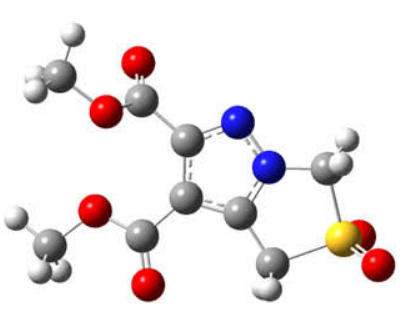

GC $(2.11)$

$\mathrm{D}_{\mathrm{Phi}}=59.2^{\circ} ; \mathrm{D}_{\mathrm{Psi}}=10.4^{\circ}$

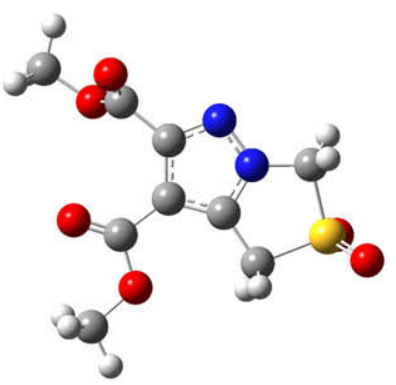

G'T (3.40)

$\mathrm{D}_{\text {Phi }}=-63.7^{\circ} ; \mathrm{D}_{\mathrm{Psi}}=171.1^{\circ}$

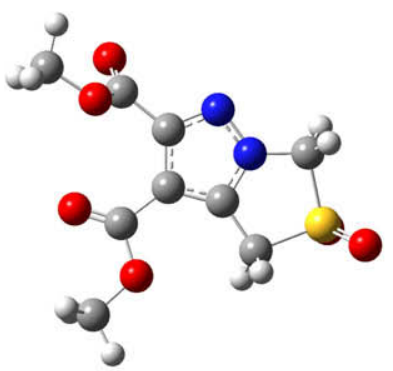

GT' (3.57)

$$
\mathrm{D}_{\mathrm{Phi}}=60.2^{\circ} ; \mathrm{D}_{\mathrm{Psi}}=-169.2^{\circ}
$$

Fig. 3. DFT(B3LYP)/6-311++G(d,p) calculated minima for conformers of DPTD. The values of zero-point corrected energy (in $\mathrm{kJ}$ mol ${ }^{-1}$ ) relative to conformer $\mathrm{S}^{\prime} \mathrm{C}$ are given in parentheses. Dihedral angles $\mathrm{N}_{28} \mathrm{C}_{4} \mathrm{C}_{15}=\mathrm{O}_{18}$ (Phi) and $\mathrm{C}_{2} \mathrm{C}_{1} \mathrm{C}_{14}=\mathrm{O}_{16}$ (Psi) are also provided.

Table 1

Calculated relative energies $\left(\Delta E\right.$ in $\mathrm{kJ} \mathrm{mol}^{-1}$ ) and dipole moments ( $\mu$ in Debyes) of the PES minima of DPTD.

\begin{tabular}{llll}
\hline Minimum & $\Delta E$ & $\Delta E_{\mathrm{ZPVE}^{\mathrm{a}}}$ & $\mu(\mathrm{D})$ \\
\hline $\mathrm{S}^{\prime} \mathrm{C}$ & 0.00 & 0.00 & 3.51 \\
$\mathrm{SC}^{\prime}$ & 0.78 & 0.63 & 4.53 \\
$\mathrm{G}^{\prime} \mathrm{C}^{\prime}$ & 2.24 & 2.08 & 4.03 \\
$\mathrm{GC}$ & 2.24 & 2.11 & 4.85 \\
$\mathrm{G}^{\prime} \mathrm{T}$ & 3.84 & 3.40 & 2.88 \\
$\mathrm{GT}^{\prime}$ & 4.04 & 3.57 & 4.27
\end{tabular}

${ }^{a}$ Relative energies including zero point energy corrections.

dihedrals, three types of minima can be defined: skew/cis ( $\mathrm{S}^{\prime} \mathrm{C}$ and $\left.\mathrm{SC}^{\prime}\right)$, gauche/cis ( $\mathrm{GC}$ and $\mathrm{G}^{\prime} \mathrm{C}^{\prime}$ ) and gauche/trans $\left(\mathrm{G}^{\prime} \mathrm{T}\right.$ and $\left.\mathrm{GT}^{\prime}\right)$. It should be emphazised that the pair of minima forming a given type of family according to the above classification is not mirror-image because the non-planarity of the thiazolidine ring. Nevertheless, these minima were found to have very similar energies $(0.0 / 0.6$, $2.1 / 2.1$ and $3.4 / 3.6 \mathrm{~kJ} \mathrm{~mol}^{-1}$, for $\mathrm{S}^{\prime} \mathrm{C} / \mathrm{SC}^{\prime}, \mathrm{GC} / \mathrm{G}^{\prime} \mathrm{C}^{\prime}$ and $\mathrm{G}^{\prime} \mathrm{T} / \mathrm{GT}^{\prime}$, respectively), reflecting their close structural similarity.

The most significant factor that determines the relative stability of the DPTD minima is the steric repulsion between the ester groups, in particular those between the lone electron pairs of the oxygen atoms. For the lowest energy $\mathrm{S}^{\prime} \mathrm{C} / \mathrm{SC}^{\prime}$ (skew/cis) structures, only one lone-electron pairs' repulsive interaction exists $\left[\mathrm{O}_{17} \cdots \mathrm{O}_{18}\right.$ $(\sim 300 \mathrm{pm})]$. For the higher energy forms, two oxygen-oxygen lone-electron pairs' repulsions are significant: $\mathrm{O}_{17} \cdots \mathrm{O}_{19}(\sim 305$ $\mathrm{pm})$ and $\mathrm{O}_{17} \cdots \mathrm{O}_{18}(\sim 375 \mathrm{pm})$, for $\mathrm{GC} / \mathrm{G}^{\prime} \mathrm{C}^{\prime}$ (gauche/cis), and $\mathrm{O}_{16} \cdots \mathrm{O}_{19}$ ( $\sim 309 \mathrm{pm}$ ) and $\mathrm{O}_{16} \cdots \mathrm{O}_{18}(\sim 385 \mathrm{pm})$, for $\mathrm{G}^{\prime} \mathrm{T} / \mathrm{GT}^{\prime}$ (gauche/trans). The slightly larger higher energies predicted for $\mathrm{G}^{\prime} \mathrm{T}$ and $\mathrm{GT}^{\prime}$ can be accounted by the fact that in these forms it is the more negative carbonyl oxygen atom $\left(\mathrm{O}_{16}\right)$ that is closest to the second carboxylic group, whereas in the $\mathrm{GC}$ and $\mathrm{G}^{\prime} \mathrm{C}^{\prime}$ forms it is the ester $\mathrm{O}_{17}$ atom, which bears a smaller negative charge. Another factor contributing to the relative stability of the DPTD minima is the repulsive interaction between $\mathrm{O}_{18}$ (one of the carbonyl oxygen atoms; see Fig. 1) and the nitrogen atom of the pyrazole ring $\left(\mathrm{N}_{28}\right)$. Indeed, in $\mathrm{S}^{\prime} \mathrm{C} / \mathrm{SC}^{\prime}$, the $\mathrm{N}_{28} \cdots \mathrm{O}_{18}(\sim 344 \mathrm{pm})$ repulsive interaction is weaker than those existing in the other pairs of structures: $\mathrm{GC} / \mathrm{G}^{\prime} \mathrm{C}^{\prime}, \mathrm{N}_{28} \cdots \mathrm{O}_{18}(\sim 303$ $\mathrm{pm})$, and $\mathrm{G}^{\prime} \mathrm{T} / \mathrm{GT}^{\prime}, \mathrm{N}_{28} \cdots \mathrm{O}_{18}(\sim 303 \mathrm{pm})$. However, this repulsive interaction is partially compensated in energetic grounds by a more important repulsive interaction between $\mathrm{N}_{28}$ and $\mathrm{O}_{19}$ in the $\mathrm{S}^{\prime} \mathrm{C} / \mathrm{SC}^{\prime}$ forms $(\sim 280 \mathrm{pm})$ than in the remaining forms $\left(\mathrm{GC} / \mathrm{G}^{\prime} \mathrm{C}^{\prime}\right.$ : $\left.\sim 332 \mathrm{pm} ; \mathrm{G}^{\prime} \mathrm{T} / \mathrm{GT}^{\prime}: \sim 333 \mathrm{pm}\right)$.

\subsection{Vibrational spectra of matrix-isolated DPTD}

In order facilitate the interpretation of the infrared spectra of matrix-isolated DPTD, it is very important to know the landscape of the PES of the molecule in the relevant regions, in particular the energy barriers for conformational interconversion between the low energy conformers. Indeed, the calculated energy barriers for conformational isomerization can be expected to be a good approximation to the real isomerization barriers of the DPTD molecule in cryogenic matrices. Though very frequently these energy barriers are large enough to allow the gas phase equilibrium conformational population to be efficiently trapped in the cryogenic matrices, when they are very low (lower than a few $\mathrm{kJ} \mathrm{mol}^{-1}$ ) the situation is considerably more complex. In this case, the energy barriers can easily be surpassed during deposition of the matrix and higher energy conformers may relax into lower energy conformers. This phenomenon known as conformational cooling is quite frequent and has been discussed in detail in some of our previous studies [35-38]. Its main consequence is to make the abundances of conformers in a matrix different from those existing in equilibrium in the gas phase prior to matrix deposition.

The B3LYP/6-311++G(d,p) calculated conformational isomerization energy barriers are given in Table 2 . The locations of the different transition states (Figure S1; Supporting Information) on the 
Table 2

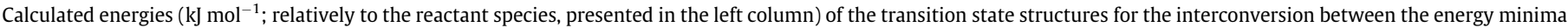
on the DFT(B3LYP)/6-311++G(d,p) potential energy surface of DPTD ${ }^{a}$.

\begin{tabular}{|c|c|c|c|c|c|c|}
\hline & $\mathrm{S}^{\prime} \mathrm{C}$ & $\mathrm{SC}^{\prime}$ & GC & $\mathrm{G}^{\prime} \mathrm{C}^{\prime}$ & $\mathrm{G}^{\prime} \mathrm{T}$ & $\mathrm{GT}^{\prime}$ \\
\hline$S^{\prime} \mathrm{C}$ & - & $7.03(6.62)$ & $2.37(1.88)$ & - & $14.95(14.65)$ & - \\
\hline $\mathrm{SC}^{\prime}$ & $6.25(5.99)$ & - & - & $1.90(1.65)$ & - & $13.76(13.70)$ \\
\hline GC & $0.13(-0.21)$ & - & - & $10.76(10.80)$ & - & $16.95(16.68)$ \\
\hline$G^{\prime} C^{\prime}$ & - & $0.44(0.17)$ & $10.77(10.77)$ & - & $16.45(16.00)$ & - \\
\hline $\mathrm{G}^{\prime} \mathrm{T}$ & $11.11(11.25)$ & - & - & $14.85(14.72)$ & - & 11.11 (10.94) 10.74 \\
\hline $\mathrm{GT}^{\prime}$ & - & $10.50(10.77)$ & $15.16(15.20)$ & - & 10.91 (10.77) 10.55 (10.58) & \\
\hline
\end{tabular}

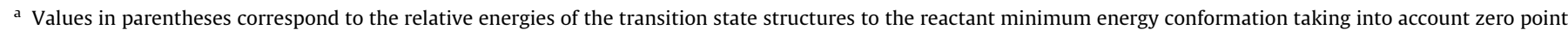
energy corrections.

PES are shown in Fig. 2, which also shows the positions of the 6 low energy minima. The transition states TS_G and TS_H are associated with the interconversion of conformers gauche/cis into conformers skew/cis. They define the energy barriers for the GC $\rightarrow S^{\prime} C$ and $\mathrm{G}^{\prime} \mathrm{C}^{\prime} \rightarrow \mathrm{SC}^{\prime}$ conversions, which were found to be extremely low: 0.13 and $0.44 \mathrm{~kJ} \mathrm{~mol}^{-1}$, respectively $(2.37$ and $1.90 \mathrm{~kJ} \mathrm{~mol} \mathrm{in} \mathrm{the} \mathrm{re-}$ verse direction). In fact, when zero point energy corrections were considered, the $\mathrm{G}^{\prime} \mathrm{C}^{\prime} \rightarrow \mathrm{SC}^{\prime}$ energy barrier reduces to only $0.17 \mathrm{~kJ} \mathrm{~mol}^{-1}$ and the $\mathrm{GC} \rightarrow \mathrm{S}^{\prime} \mathrm{C}$ process becomes barrierless, with the energy of TS_G being $0.21 \mathrm{~kJ} \mathrm{~mol}^{-1}$ below that of the GC form. In practical terms, this means that GC shall not be considered a real conformer, but a vibrationally excited form of conformer $S^{\prime} C$. On the other hand, it can be also anticipated that during matrix deposition the $\mathrm{G}^{\prime} \mathrm{C}^{\prime}$ conformer should relax promptly to conformer $\mathrm{SC}^{\prime}$. Since as detailed below all the other relevant energy barriers for conformational isomerization are large enough to prevent any conversion to take place during matrix deposition, it can be concluded that two types of conformers, skew/cis $\left(\mathrm{S}^{\prime} \mathrm{C} / \mathrm{SC}^{\prime}\right)$ and gauche/trans $\left(\mathrm{G}^{\prime} \mathrm{T} / \mathrm{GT}^{\prime}\right)$, are then expected to be present in the matrices. Conformer $\mathrm{SC}^{\prime}$ should have a population given by the sum of its gas phase population at the temperature of deposition with that of the $G^{\prime} C^{\prime}$ form. The estimated populations for the four experimentally relevant conformers (in matrices: $\mathrm{S}^{\prime} \mathrm{C}$ : $\mathrm{SC}^{\prime}: \mathrm{G}^{\prime} \mathrm{T}$ : $\mathrm{GT}^{\prime}$ ), at the temperatures used in the experiments undertaken in this study, are $33 \%$ : $44 \%$ : $11 \%$ : $11 \%$ (within an unit of percentage, the populations are identical at 383 and $403 \mathrm{~K}$, the two experimentally relevant temperatures; see Section 2).

As mentioned above, all other relevant barriers to conformational isomerization in DPTD are considerably larger than those between the gauche/cis and skew/cis structures. The $\mathrm{SC}^{\prime} \rightarrow \mathrm{S}^{\prime} \mathrm{C}$ isomerization (between the two skew/cis conformers; associated with transition state TS_F) has a barrier of $5.99 \mathrm{~kJ} \mathrm{~mol}^{-1}$ (6.62 $\mathrm{kJ} \mathrm{mol}^{-1}$ in the reverse direction), whereas that interconverting $\mathrm{GC}$ and $\mathrm{G}^{\prime} \mathrm{C}^{\prime}$ (the two gauche/cis structures; TS_E) amounts to $c a$. $10.8 \mathrm{~kJ} \mathrm{~mol}^{-1}$ in both directions. In turn, the predicted energy barriers for conversion between gauche/trans conformers $\left(\mathrm{GT}^{\prime} \rightarrow \mathrm{G}^{\prime} \mathrm{T}\right)$ are 10.58 and $10.77 \mathrm{~kJ} \mathrm{~mol}^{-1}\left(10.75\right.$ and $10.94 \mathrm{~kJ} \mathrm{~mol}^{-1}$ in the reverse direction), corresponding to two different reaction pathways (associated with transition states TS_C and TS_D, respectively; see Fig. 2). The pathway for conversion of conformers gauche/trans into skew/cis (i.e., $\mathrm{G}^{\prime} \mathrm{T} \rightarrow \mathrm{S}^{\prime} \mathrm{C}$ and $\mathrm{GT}^{\prime} \rightarrow \mathrm{SC}^{\prime}$ ) have energy barriers dictated by the transitions states TS_I and TS_J, with values of 11.25 and $10.77 \mathrm{~kJ} \mathrm{~mol}^{-1}$, respectively. TS_A and TS_B could also in principle allow the interconversion of forms gauche/trans into less energetic conformers. However, the corresponding barriers are considerably larger $\left(\sim 15 \mathrm{~kJ} \mathrm{~mol}^{-1}\right)$ than those corresponding to alternative pathways, which make these processes of no interest in practical terms.

It shall be noticed that all the energy barriers now discussed are only moderately high (less than $c a .10 \mathrm{~kJ} \mathrm{~mol}^{-1}$ ) and, then, conversions of the gauche/trans conformers into the skew/cis forms, as well as between the higher energy member of each family of con- formers into the lowest energy one, could also in principle take place upon annealing of the matrices to higher temperatures. We shall turn to this point below.

The spectra of DPTD isolated in $\operatorname{argon}(T=10 \mathrm{~K})$ and xenon $(T=20 \mathrm{~K})$ matrices are presented in Fig. 4. All four relevant conformers of DPTD belong to the $C_{1}$ symmetry point group, with 78 fundamental vibrations, all of them expected to be active in the infrared. The calculated spectra for these conformers and the corresponding results of normal coordinates analysis are provided as Supporting Information (Tables S2-S6). Since the two fused rings in the molecule are not significantly affected by the changes in orientation of the ester groups, their associated vibrations are similar and give rise to bands practically coincident in all conformers. In addition, within each family of conformers $\left(\mathrm{S}^{\prime} \mathrm{C} / \mathrm{SC}^{\prime}\right.$ and $\left.\mathrm{G}^{\prime} \mathrm{T} / \mathrm{GT}^{\prime}\right)$ the ester fragments were also found to give rise to practically coincident spectra. On the other hand, some of these vibrations

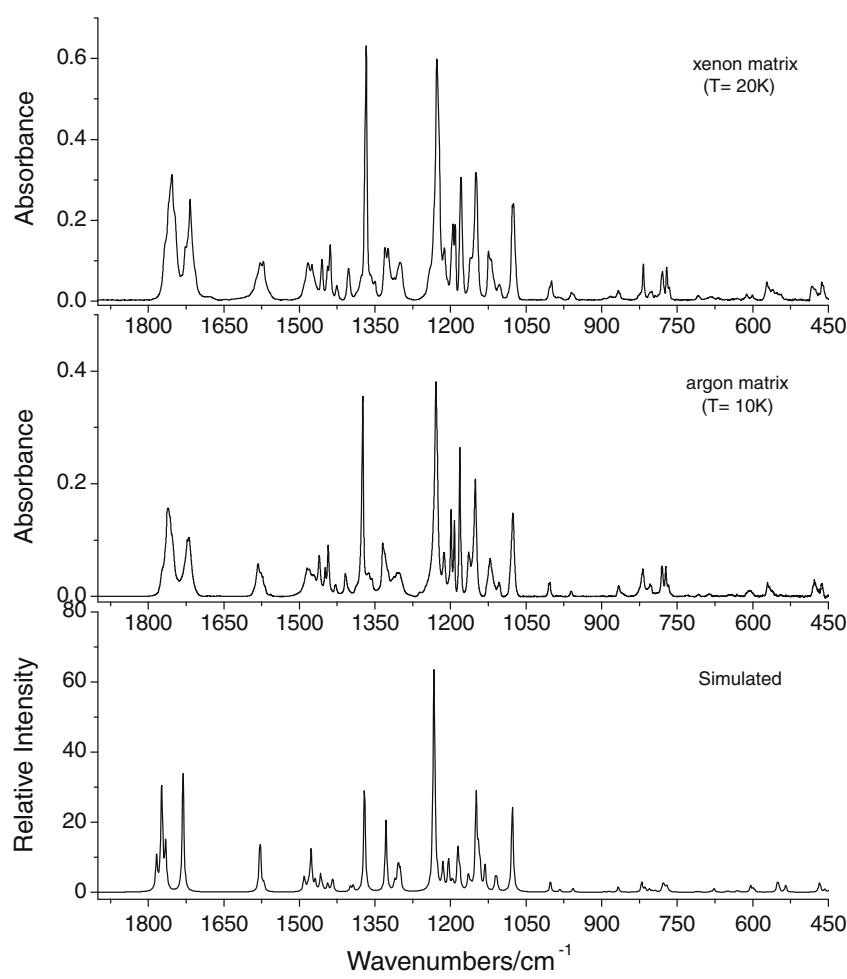

Fig. 4. Infrared spectra of DPTD isolated in solid argon and xenon (as-deposited matrices; substrate temperatures: xenon, $20 \mathrm{~K}$; argon, $10 \mathrm{~K}$ ) and simulated spectrum built by adding the DFT(B3LYP)/6-311++G(d,p) calculated spectra of conformers $\mathrm{S}^{\prime} \mathrm{C}, \mathrm{SC}^{\prime}, \mathrm{G}^{\prime} \mathrm{T}$ and $\mathrm{GT}^{\prime}$ scaled by their relative populations at the sublimation temperatures (estimated from calculated relative energies and assuming the Boltzmann distribution; see text). The theoretical spectra were simulated using Lorenzian functions centered at the calculated frequencies and with a bandwidthat-half-height equal to $4 \mathrm{~cm}^{-1}$. 
are sufficiently different in the two families and could be used successfully for their spectroscopic identification.

The theoretically simulated spectrum, obtained by taking into account the calculated spectra for conformers $S^{\prime} C, S C^{\prime}, G^{\prime} T$ and $\mathrm{GT}^{\prime}$ weighted by their expected populations in the as-deposited matrices, is also shown in Fig. 4, for comparison. As it can be seen, the experimental spectra of DPTD in both argon and xenon matrices are well reproduced by the simulated spectrum.

Identification of bands due to each family of conformers (skew/ cis and gauche/trans) would be relatively easy if we could change their population in the matrix. This could in principle be done in two ways: varying the temperature of the gas phase prior to deposition, or taking advantage of conformational cooling during deposition of the matrix or upon annealing of the deposited matrix to higher temperatures. Very unfortunately, none of these strategies were found to be successfully in this case. Changing the temperature of the gas prior to deposition was not efficient, since within the usable temperature range (from the temperature at which the compound starts to sublimate to that at which the compound starts to decompose, i.e., from $\mathrm{ca}$. $360 \mathrm{~K}$ to $c a .450 \mathrm{~K}$ ) the change in populations are too small (less than $\mathrm{ca}$. $3 \%$ ) to be noticeable. On the other hand annealing of the argon matrices up to the maximum possible temperature (ca. $35 \mathrm{~K}$; from that on, the argon matrix starts to evaporate considerably and becomes unusable) was found not to be enough to provide the required energy for the isomerization reactions to occur and the spectrum did not change. In turn, the annealing experiments performed in the xenon matrices (up to $75 \mathrm{~K}$ ) lead to spectral changes for the high temperatures investigated, but these are mainly ascribable to aggregation. Even if it took place simultaneously with aggregation, conformational isomerization could not be spectroscopically proved without any doubt. Furthermore, the experiments in which the temperature of the substrate was increased during deposition of the xenon matrices led essentially to the same results as the annealing experiments in the same type of matrix. Hence, the identification of the two types of conformers of DPTD in the matrices had to be done by simple direct comparison between the experimental spectra and their calculated spectra. Fortunately, there are a few spectral regions where the bands due to the two types of conformers are predicted to appear enough well separated. Figs. 5 and 6 show the $750-850 \mathrm{~cm}^{-1}$ and $1050-1275 \mathrm{~cm}^{-1}$ regions, where bands ascribable to both types of experimentally relevant conformers can be observed. In these figures, the simulated spectra correspond to the sum of the calculated spectra of the two conformers belonging to the same family (dashed lines: $\mathrm{S}^{\prime} \mathrm{C}$ and $\mathrm{SC}^{\prime}$; solid lines: $\mathrm{G}^{\prime} \mathrm{T}$ and $\mathrm{GT}^{\prime}$ ) with the intensities weighted by their expected populations in the matrices.

In the low frequency range (Fig. 5), the intense bands around $820 \mathrm{~cm}^{-1}$ and the two main bands in the $785-770 \mathrm{~cm}^{-1}$ range are essentially due to the $\mathrm{S}^{\prime} \mathrm{C}$ and $\mathrm{SC}^{\prime}$ forms, the first group of bands being mainly due to the $\delta \mathrm{OCO}$ and $\delta$ (T-ring 2 ) modes and the second to $\delta \mathrm{OCO}^{\prime}$ and the two $\gamma \mathrm{C}=\mathrm{O}$ rocking modes (Tables S3 and S4). On the other hand, the lower intensity bands in the $805-790 \mathrm{~cm}^{-1}$ region and at ca. $766 \mathrm{~cm}^{-1}$, as well as the shoulder ca. $775 \mathrm{~cm}^{-1}$ in the spectra of the xenon matrix are mainly due to the $\mathrm{G}^{\prime} \mathrm{T}$ and $\mathrm{GT}^{\prime}$ conformers and ascribable to vibrations involving predominantly the $\delta \mathrm{OCO}$ and $\gamma \mathrm{C}=\mathrm{O}$ coordinates (Tables S5 and S6).

In the higher frequency region (Fig. 6) there is a more pronounced overlap between the bands originated in the two pairs of conformers. However, the bands at ca. 1215 and $1125 \mathrm{~cm}^{-1}$ (in particular the one at $1215 \mathrm{~cm}^{-1}$ ) can be safely taken as being originated mostly in the less populated $\mathrm{G}^{\prime} \mathrm{T}$ and $\mathrm{GT}^{\prime}$ conformers, the coupled $\gamma \mathrm{CH}_{3}(2) / \nu \mathrm{C}-\mathrm{O}^{\prime}$ and $v(\mathrm{~N}-\mathrm{N}$ P-ring $) / \mathrm{twCH}_{2}$ vibrations being the dominating contributors to these bands, respectively (see Tables S5 and S6).

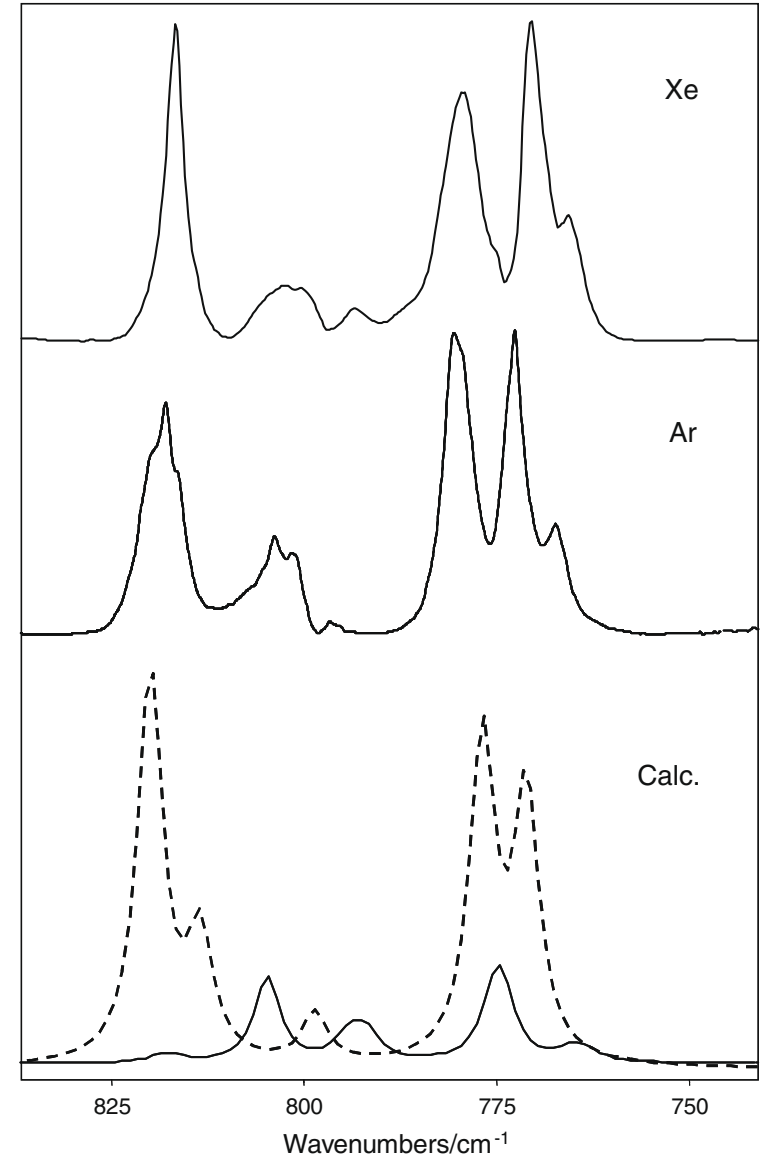

Fig. 5. Experimental spectra $\left(750-850 \mathrm{~cm}^{-1}\right.$ range) of DPTD isolated in xenon $(20 \mathrm{~K})$ and argon $(10 \mathrm{~K})$ matrices, and simulated spectra constructed from the DFT(B3LYP)/6-311++G(d,p) calculated spectra of the two pairs of conformers (dashed lines: $\mathrm{S}^{\prime} \mathrm{C}$ and $\mathrm{SC}^{\prime}$; solid lines: $\mathrm{G}^{\prime} \mathrm{T}$ and $\mathrm{GT}^{\prime}$ ). In the simulated spectra, the intensities of the calculated spectra of individual conformers were weighted by their expected populations in the matrices $\left(\mathrm{S}^{\prime} \mathrm{C}: \mathrm{SC}^{\prime}: \mathrm{G}^{\prime} \mathrm{T}: \mathrm{GT}^{\prime}=33 \%: 44 \%\right.$ : $11 \%$ : $11 \%$; see text).

The full list of observed bands both in argon and xenon matrices and the proposed assignments are summarized in Table S7.

\subsection{Vibrational spectra of neat condensed phases of DPTD and of DPTD in $\mathrm{KBr}$ pellet}

Figure S2 (Supporting Information) displays the infrared spectra of DPTD in the neat condensed phases and in $\mathrm{KBr}$ pellet: polycrystalline DPTD in a $\mathrm{KBr}$ pellet, at room temperature (298 K); melted DPTD in $\mathrm{KBr}$ pellet (418 K); low temperature (20 K) amorphous phase resulting from fast deposition of the vapour (at $403 \mathrm{~K}$ ) of the compound onto the cold substrate of the cryostat and crystalline state resulting from warming the previous sample at $298 \mathrm{~K}$ and subsequently cooling it to $20 \mathrm{~K}$. As seen in this Figure, the room temperature spectrum of the polycrystalline DPTD in a $\mathrm{KBr}$ pellet is identical to the spectrum of the crystalline form obtained by heating the amorphous film resulting from deposition of the vapour of the compound at low temperature, despite this latter exhibits the expected band narrow effect due to the low temperature. Also the spectrum of the neat low temperature amorphous state and melted phase in $\mathrm{KBr}$ pellet are similar. Though a definitive answer to the conformation assumed by DPTD in the crystal should rely on X-ray data, the comparison of spectrum of the crystal with those calculated for the different conformers of DPTD strongly suggests that in the crystalline state the DPTD molecules exist in a skew/cis conformation. In Fig. 7, the $750-850 \mathrm{~cm}^{-1}$ spec- 


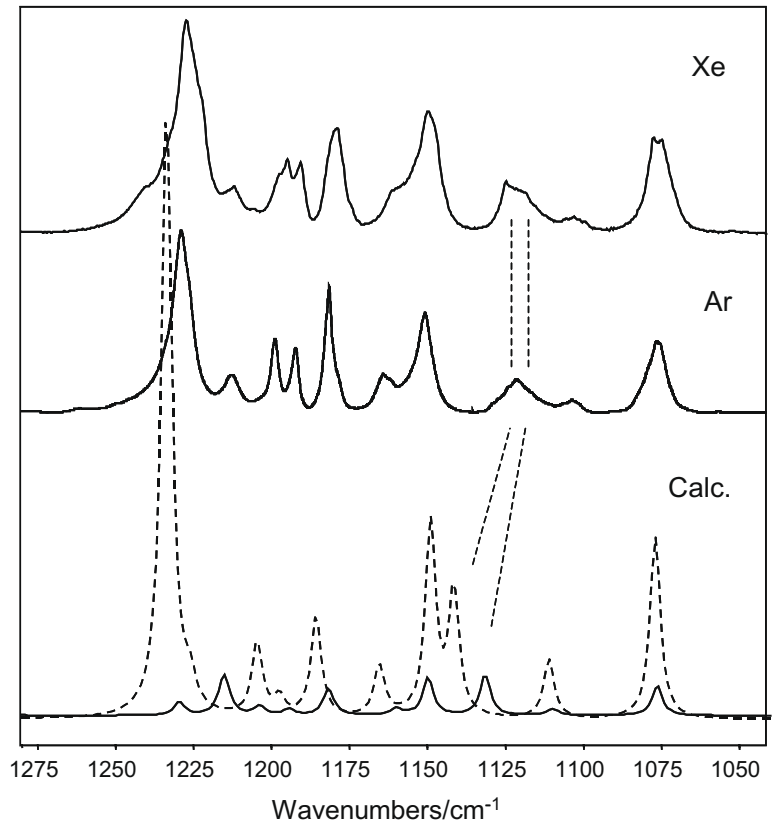

Fig. 6. Experimental spectra (1275-1050 $\mathrm{cm}^{-1}$ range) of DPTD isolated in xenon $(20 \mathrm{~K})$ and argon $(10 \mathrm{~K})$ matrices, and simulated spectra constructed from the DFT(B3LYP)/6-311++G(d,p) calculated spectra of the two pairs of conformers (dashed lines: $\mathrm{S}^{\prime} \mathrm{C}$ and $\mathrm{SC}^{\prime}$; solid lines: $\mathrm{G}^{\prime} \mathrm{T}$ and $\mathrm{GT}^{\prime}$ ). In the simulated spectra, the intensities of the calculated spectra of individual conformers were weighted by their expected populations in the matrices $\left(\mathrm{S}^{\prime} \mathrm{C}: \mathrm{SC}^{\prime}: \mathrm{G}^{\prime} \mathrm{T}: \mathrm{GT}^{\prime}=33 \%: 44 \%: 11 \%: 11 \%\right.$; see text).

tral range of the spectrum of the crystal in $\mathrm{KBr}$ pellet is shown in detail (top panel, dashed line). In this spectrum, the characteristic bands of the gauche/trans forms in the $805-790 \mathrm{~cm}^{-1}$ region and at ca. $766 \mathrm{~cm}^{-1}$ and $775 \mathrm{~cm}^{-1}$ are absent. Upon increasing the temperature of the $\mathrm{KBr}$ pellet to $145^{\circ} \mathrm{C}(418 \mathrm{~K})$, the melt is obtained, the phase transition being easily observed spectroscopically. Particularly interesting is the appearance of new bands in the spectrum, in particular those that are characteristic of the gauche/ trans conformers, which doubtlessly indicate that in this phase DPTD exists as a mixture of different conformers. The 750$850 \mathrm{~cm}^{-1}$ spectral range, shown in Fig. 7, clearly reveals the presence of both skew/cis and gauche/trans conformers in the melted state, through simple comparison with the calculated spectra for these types of conformers that are also presented in the figure, for comparison. The calculated spectra were simulated summing the theoretically obtained spectra for the conformers of each family (dashed line: $\mathrm{S}^{\prime} \mathrm{C}$ and $\mathrm{SC}^{\prime}$; thick solid line: $\mathrm{G}^{\prime} \mathrm{T}$ and $\mathrm{GT}^{\prime}$ ) with the intensities weighted by their expected populations at $418 \mathrm{~K}$. Note that in the melted state, we could also expect a contribution from conformer $\mathrm{G}^{\prime} \mathrm{C}^{\prime}$, since no conformational cooling can take place in this case. However, considering the similarity of the spectrum of this conformer and those of the gauche/trans conformers (see Fig. 7) it is not possible to confirm this in a definitive way. It shall be noticed that in the spectrum of the low temperature amorphous neat compound, conformational cooling is expected to take place and in this case only the skew/cis and gauche/trans conformers shall contribute to the spectrum. Taking this into account, comparison of the spectra of the neat amorphous state and melted phase could, in principle, give us some indication of the features in this latter spectrum that are given rise by the $\mathrm{G}^{\prime} \mathrm{C}^{\prime}$ form. However, the bands in both spectra are considerably broad and since, as already mentioned, the spectrum of the $\mathrm{G}^{\prime} \mathrm{C}^{\prime}$ conformer is very similar to those of the gauche/trans conformers, it was not possible in practice to identify with certainty any band in the spectrum of the melt that can be ascribed to the $\mathrm{G}^{\prime} \mathrm{C}^{\prime}$ form.

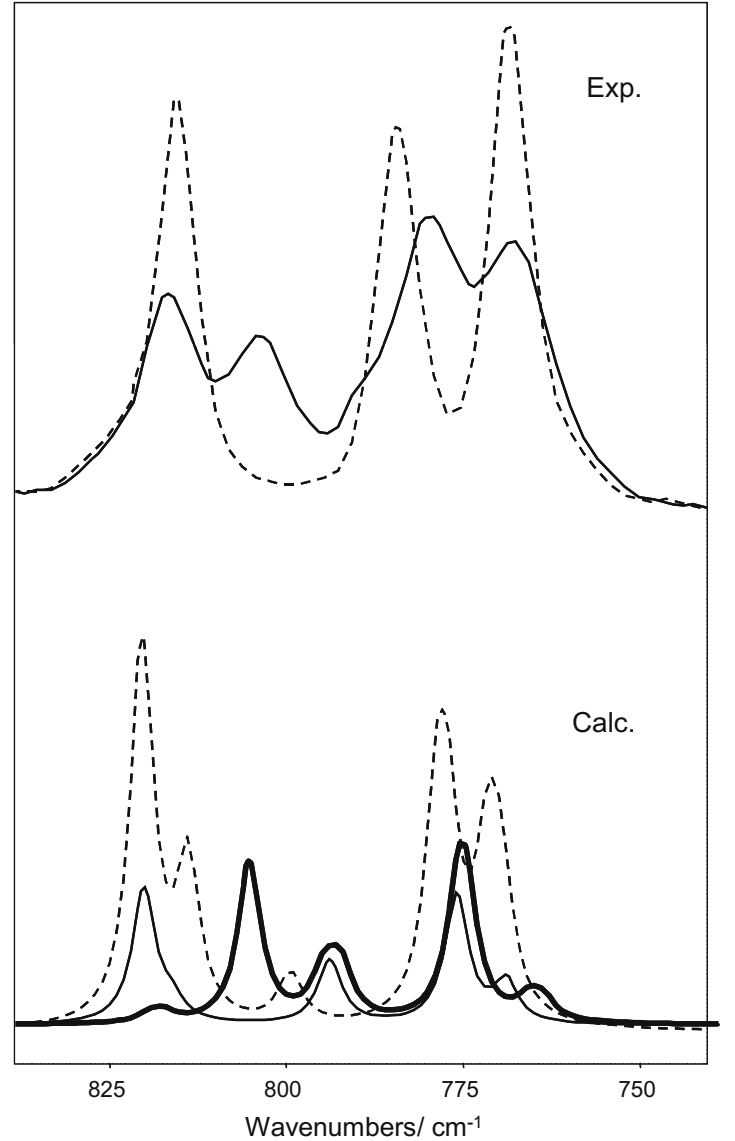

Fig. 7. Top: experimental infrared spectra $\left(750-850 \mathrm{~cm}^{-1}\right.$ range) of DPTD in $\mathrm{KBr}$ pellet: polycrystalline, at room temperature $(298 \mathrm{~K})$ - dashed line; melted phase $(418 \mathrm{~K})$ - solid line; Bottom: simulated spectra obtained by summing the theoretically obtained spectra for the conformers of each family (dashed line: $\mathrm{S}^{\prime} \mathrm{C}$ and $\mathrm{SC}^{\prime}$; thick solid line: $\mathrm{G}^{\prime} \mathrm{T}$ and $\mathrm{GT}^{\prime}$; thin solid line: $\mathrm{G}^{\prime} \mathrm{C}^{\prime}$ ) with the intensities weighted by their expected populations at $418 \mathrm{~K}$ according to the calculations $\left(S^{\prime} \mathrm{C}: S C^{\prime}: G^{\prime} T: G T^{\prime}: G^{\prime} C^{\prime}=32: 27: 18: 12: 11 \%\right)$.

Table S8 (Supporting Information) presents the proposed assignments for the spectra obtained in $\mathrm{KBr}$ pellet (for both crystal a melted states) and for the low temperature neat amorphous and crystalline phases.

\section{Conclusions}

The pyrazolo-thiazole precursor of extended dipole diazafulvenium methide intermediates, dimethyl 2,2-dioxo- $1 H, 3 H$-pyrazolo[1,5-c][1,3]thiazole-6,7-dicarboxylate, DPTD, was studied by infrared spectroscopy under different experimental conditions (in both argon and xenon matrices, as a polycrystalline sample and melted phase in $\mathrm{KBr}$ pellet, and in the neat amorphous and crystalline solid states). The experimental data was interpreted taking into account results obtained by quantum chemical calculations performed at the DFT/B3LYP level of theory using both the 6$31+G(d)$ and $6-311++G(d, p)$ basis sets. Six low energy minima were identified in the resulting potential energy surfaces $\left(S^{\prime} C, S C^{\prime}, G C\right.$, $\mathrm{G}^{\prime} \mathrm{C}^{\prime}, \mathrm{G}^{\prime} \mathrm{T}$ and $\mathrm{GT}^{\prime}$ ) with relative energies within $\mathrm{ca} .4 .5 \mathrm{~kJ} \mathrm{~mol}^{-1}$. Consideration of zero-point energies and barriers to conformational isomerization; however, led to establish the practical relevance of only two pairs of conformers for the compound under the low temperature experimental conditions considered in this investigation: skew/cis (conformers $\mathrm{S}^{\prime} \mathrm{C}$ and $\mathrm{SC}^{\prime}$ ) and gauche/trans (conformers $\mathrm{G}^{\prime} \mathrm{T}$ and $\mathrm{GT}^{\prime}$ ). 
In consonance with the theoretical analysis of the potential energy surface of DPTD, the detailed analysis of the matrix-isolated DPTD infrared spectra reveal the presence in the matrices of both skew/cis and gauche/trans conformers. The same conformers of DPTD were also found in both the amorphous phase resulting from fast deposition of the sole vapour of the compound onto the cold $(20 \mathrm{~K})$ substrate of the cryostat and in the melted compound at high temperature. In this latter phase, the presence of the $\mathrm{G}^{\prime} \mathrm{C}^{\prime}$ conformer could not be neither proved nor excluded, though in view of the theoretical results its presence in this phase appears to be quite probable. On the other hand, in the neat crystalline state the vibrational signature of the compound indicates that it exists only in a skew/cis arrangement.

\section{Acknowledgements}

This work has been funded by the Portuguese Science Foundation (FCT, Lisbon), under research project PTDC/QUI/71203/2006. S.L. and C.M.N. also thank FCT for the Ph.D. Grants \#SFRH/BD/ 29698/2006 and \#SFRH/BD/28844/2006.

\section{Appendix A. Supplementary data}

Supplementary data associated with this article can be found, in the online version, at doi:10.1016/j.molstruc.2008.12.061.

\section{References}

[1] T.R. Gilchrist, Heterocyclic Chemistry, third ed., Longman, Essex, 1997.

[2] T.M.V.D. Pinho e Melo, M.I.L. Soares, A. M. d'A. Rocha Gonsalves, H. McNab, Tetrahedron Lett. 45 (2004) 3889.

[3] T.M.V.D. Pinho e Melo, Maria I.L. Soares, A.M.d'A. Rocha Gonsalves, J.A. Paixão, A. Matos Beja, M. Ramos Silva, J. Org. Chem. 70 (2005) 6629.

[4] T.M.V.D. Pinho e Melo, M.I.L. Soares, A.M.d'A. Rocha Gonsalves, Tetrahedron Lett. 47 (2006) 791

[5] T.M.V.D. Pinho e Melo, M.I.L. Soares, C.M. Nunes, Tetrahedron 63 (2007) 1833

[6] T.M.V.D. Pinho e Melo, C.M. Nunes, M.I.L. Soares, J.A. Paixão, A. Matos Beja, M. Ramos Silva, J. Org. Chem. 72 (2007) 4406.

[7] M.I.L. Soares, T.M.V.D. Pinho e Melo, Tetrahedron Lett. 49 (2008) 4889.

[8] O.B. Sutcliffe, R.C. Storr, T.L. Gilchrist, P. Rafferty, A.P.A. Crew, Chem. Comm. (2000) 675.

[9] O.B. Sutcliffe, R.C. Storr, T.L. Gilchrist, P. Rafferty, Tetrahedron 56 (2000) 10011.

[10] J.M. Kane, J. Org. Chem. 45 (1980) 5396.

[11] W.K. Anderson, R.H. Mach, J. Med. Chem. 30 (1987) 2109

[12] D. Ladureé, J.-C. Lancelot, M. Robba, E. Chenu, G. Mathé, J. Med. Chem. 32 (1989) 456.
[13] D. Lavé, C. James, H. Rajoharison, P.E. Bost, I. Cavero, Drugs Future 14 (1989) 891.

[14] J. Fabre, D. Farge, C. James, D. Lavé, US Patent 4529728, 1985.

[15] Fabre, J.; James, C.; Lavé, D. US Patent 4786645, 1988.

[16] S.K. Davidsen, J.B. Summers, D.H. Albert, J.H. Holms, H.R. Heyman, T.J. Magoc, R.G. Conway, D.A. Rhein, G.W. Carter, J. Med. Chem. 37 (1994) 4423.

[17] A. Kaczor, T.M.V.D. Pinho e Melo, Maria I.L. Soares, R. Fausto, J. Phys. Chem. A 110 (2006) 6531-6539.

[18] Frisch, M.J.; Trucks, G. W.; Schlegel, H. B.; Scuseria, G. E.; Robb, M. A. Cheeseman, J. R.; Montgomery, J. A., Jr.; Vreven, T.; Kudin, K. N.; Burant, J. C.; Millam, J. M.; Iyengar, S. S.; Tomasi, J.; Barone, V.; Mennucci, B.; Cossi, M.; Scalmani, G.; Rega, N.; Petersson, G. A.; Nakatsuji, H.; Hada, M.; Ehara, M.; Toyota, K.; Fukuda, R.; Hasegawa, J.; Ishida, M.; Nakajima, T.; Honda, Y.; Kitao, O.; Nakai, H.; Klene, M.; Li, X.; Knox, J. E.; Hratchian, H. P.; Cross, J. B.; Bakken, V.; Adamo, C.; Jaramillo, J.; Gomperts, R.; Stratmann, R. E.; Yazyev, O.; Austin, A. J.; Cammi, R.; Pomelli, C.; Ochterski, J. W.; Ayala, P. Y.; Morokuma, K.; Voth, G. A.; Salvador, P.; Dannenberg, J. J.; Zakrzewski, V. G.; Dapprich, S.; Daniels, A. D.; Strain, M. C.; Farkas, O.; Malick, D. K.; Rabuck, A. D.; Raghavachari, K.; Foresman, J. B.; Ortiz, J. V.; Cui, Q.; Baboul, A. G.; Clifford, S.; Cioslowski, J.; Stefanov, B. B.; Liu, G.; Liashenko, A.; Piskorz, P.; Komaromi, I.; Martin, R. L.; Fox, D. J.; Keith, T.; Al-Laham, M. A.; Peng, C. Y.; Nanayakkara, A.; Challacombe, M.; Gill, P. M. W.; Johnson, B.; Chen, W.; Wong, M. W.; González, C.; Pople, J. A. Gaussian 03, revision B.01; Gaussian, Inc.: Wallingford, CT, 2004.

[19] M. Frisch, M. Head-Gordon, Pople, J. Chem. Phys. Lett. 166 (1990) 281.

[20] A. Becke, Phys. Rev. A 38 (1988) 3098.

[21] C. Lee, W. Yang, R. Parr, Phys. Rev. B 37 (1988) 785.

[22] A.H. Zeng, L. Yu, Y. Wang, Q.Y. Kong, Q. Xu, M.F. Zhou, J. Phys. Chem. A 108 (2004) 6656

[23] A. Borba, P. Simões, R. Fausto, J. Phys. Chem. A 109 (2005) 3578.

[24] A. Borba, A. Gómez-Zavaglia, P. Simoes, R. Fausto, Spectrochim. Acta A - Mol. Biomol. Spectrosc. 61 (2005) 1461

[25] A. Kaczor, R. Almeida, A. Gómez-Zavaglia, M.L.S. Cristiano, R. Fausto, J. Mol. Struct. 876 (2008) 77

[26] C. Peng, H.B. Schlegel, Isr. J. Chem. 33 (1994) 449.

[27] J. Schachtschneider, Technical Report; Shell Development Co.: Emeryville, CA, 1969.

[28] M. Nowak, L. Lapinski, BALGA computer program for PED calculations.

[29] P. Pulay, G. Fogarasi, F. Pang, J.E. Boggs, J. Am. Chem. Soc. 110 (1979) 2550.

[30] A. Gómez-Zavaglia, A. Kaczor, A.L. Cardoso, T.M.V.D. Pinho e Melo, R. Fausto, J Phys. Chem. A 110 (2006) 10742.

[31] A. Gómez-Zavaglia, A. Kaczor, A.L. Cardoso, T.M.V.D. Pinho e Melo, R. Fausto, J Phys. Chem. A 110 (2006) 8081

[32] E.M.S. Maçôas, L. Khriachtchev, M. Pettersson, R. Fausto, M. Räsänen, J. Phys Chem. A 109 (2005) 3617.

[33] J.-H. Lii, J. Phys. Chem. A 106 (2002) 8667.

[34] K.B. Wiberg, K.E. Laidig, J. Am. Chem. Soc. 109 (1987) 5935.

[35] I.D. Reva, S.G. Stepanian, L. Adamowicz, R. Fausto, Chem. Phys. Lett. 374 (2003) 631

[36] A. Gómez-Zavaglia, R. Fausto, Phys. Chem. Chem. Phys. 5 (2003) 52.

[37] I.D. Reva, A.J. Lopes Jesus, M.T. Rosado, R. Fausto, M.E. Eusébio, J.S. Redinha, Phys. Chem. Chem. Phys. 8 (2006) 5339.

[38] A. Borba, A. Gómez-Zavaglia, R. Fausto, J. Mol. Struct. 794 (2006) 196. 